\title{
The Study of the Magnetic Memory's Magneto-Mechanical Coupled Effect Based on ANSYS
}

\author{
Shujun $\mathrm{Liu}^{1}{ }^{1} \mathrm{a*}$, Shenglin $\mathrm{Li}^{1}$, Ming Jiang ${ }^{1}$, Dean $\mathrm{He}^{1}$ \\ ${ }^{1}$ Logistics Engineering University, Chongqing 401311, China \\ a119042856@qq.com
}

Keywords: ANSYS, Finite element simulation, Magneto-mechanical coupled effect, Metal magnetic memory testing technology

\begin{abstract}
In the paper, a magneto-mechanical coupling model is established to study the magnetic memory's magneto-mechanical coupled effect, and then the finite element analysis software ANSYS is used to simulate the relationship between the parameter of three kinds of pipeline defects and their magnetic leakage field's normal component peak-peak value. The result proves the relationship between the stress concentration and the magnetic memory effect.
\end{abstract}

\section{Introduction}

As a fast and efficient means of energy transmission, Petroleum and natural gas pipeline transportation's safe operation is also highly valued in modern society. Usually, due to inclusion and segregation, hidden discontinuous region often occur in the inside of the pipeline material. Because of the effect of load in the manufacture, installation and use of pipe, these areas will develop into a microscopic or quasi microscopic stress concentration area. As one of the main factors that cause the safety accident of pipeline, cracks, corrosion is often sprouting in these parts, gradually accumulates macro defects, and then leads to pipeline failure. It is very important to determine the stress concentration, to study the extent of the damage, quantify of defects such as cracks and corrosion parameters, and then to carry out the early diagnosis of pipeline failure and residual life prediction.

Traditional nondestructive testing methods, such as ultrasound, magnetic flux leakage, X-ray, etc, can find the macro or most obvious defects which have been developed such as Crack, fold and inclusion, etc, by testing the key parts of the material, equipment and components. However, it is difficult to realize effective prediction for the early damage of equipment and components in service. In 1997, Russian scholar Doubov first proposed the Metal Magnetic Memory Testing Technology (MMMT) [1, 2]. The technology can carry on the fast non destructive testing to the stress concentration area of the ferromagnetic component as well as the initiation and development of cracks, corrosion and other defects. As shown in Fig. 1, the basic principle of the Metal Magnetic Memory Testing technology can be expressed as: For the effect of the load, the domain organization orientation will cause magnetostriction properties and irreversible reorientation in the Ferromagnetic components working in a geomagnetic field. The maximum leakage magnetic field is formed in the stress and deformation zone. The tangential component of the magnetic field in the stress concentration area $H_{P}(x)$ has the maximum value, while the sign of the normal component $H_{P}(y)$ changes and has zero-crossing position. The irreversible change of the magnetic state remains after the elimination of the working load. By the testing of the magnetic flux leakage field's normal component $H_{P}(y)$ and the calculation of gradient, the stress concentration position of the component can be exactly deduced $[3,4]$. 


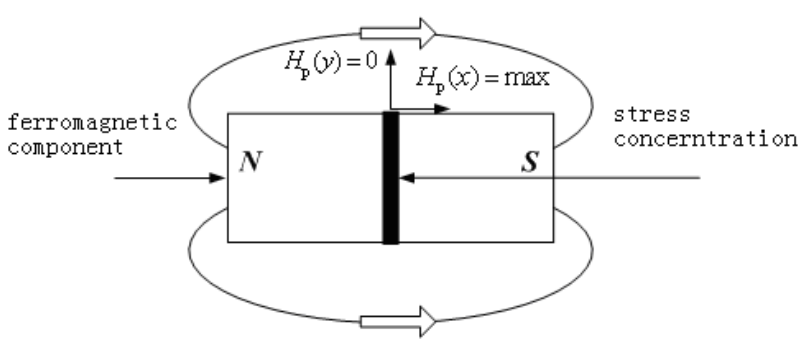

Fig. 1 Schematic of magnetic memory method.

To a certain extent, the birth of magnetic memory testing technology makes up for the deficiency of the traditional nondestructive testing methods, and the Metal Magnetic Memory Testing Technology is the only feasible nondestructive testing method in the early diagnosis of ferromagnetic metallic materials so far. Therefore, in recent years it has received extensive attention. However, the research and application of metal magnetic memory are mostly focused on the qualitative analysis of the stress concentration area. In order to clarify the relationship between the normal component and the internal stress of the magnetic flux leakage signal and advance the quantification study of magnetic memory testing, in the paper, the X60 level $\phi 150$ pipeline with defects is regarded as a model, which is widely used in oil and gas pipelines. By using the ANSYS finite element analysis software and the force magnetic coupling model, the relationship between the size of the stress concentration and the leakage magnetic field is analyzed. Simulation test is carried out for the purpose of quantifying detection of pipeline defects with MMMT.

\section{Basic Theory of Magnetic Memory's Magneto-Mechanical Coupled Effect}

When the elastic stress is acting on the ferromagnetic component, the ferromagnetic material is not only to produce elastic strain, but also the strain of the magnetostrictive property [5]. This will cause the magnetic domain wall displacement and change its spontaneous magnetization direction. We usually call this phenomenon the magneto elastic effect or the force induced contraction. The reason the magnetoelastic effect is produced is the domain structure in ferromagnet and its close relationship between stress states. After being subjected to the action of the stress, the ferromagnetic experience in the magnetic crystal body to add stress energy. According to principle of the actual state of existence must be the minimum energy state. Only by reducing the stress energy, it can make it tend to be the smallest, and make the iron magnet be in a new stable state. The way to reduce the stress energy is to change the direction of magnetization. For materials with isotropic magnetostrictive materials, when there is an external stress, applying the pulling force will make the magnetization direction of the magnetic material to be in the direction of the tension, while applying the pulling force will make the magnetization direction perpendicular to the direction perpendicular to the tensile force of the material. Under the action of stress, the magnetization vector of ferromagnetic material will be re oriented and the permeability to the stress direction can be changed. According to the basic theory of iron magnetism, a coupled model of the relationship between the change of relative permeability and the stress of ferromagnetic materials is established by Wang Sheliang [6].

$$
\frac{\Delta \mu}{\mu}=-\frac{2 \lambda_{m}}{B_{m}^{2}} \sigma \mu
$$

In the formula, $\mu$ is ferromagnetic material permeability. $\lambda_{m}$ is the ferromagnetic material saturation magnetostrictive coefficient. $\Delta \mu$ is the variation of permeability of ferromagnetic materials. $B_{m}$ is Ferromagnetic material saturation magnetic induction strength. $\sigma$ is stress. The formula (1) shows that: 
First, the relative change rate of the magnetic permeability of the ferromagnetic material $\frac{\Delta \mu}{\mu}$ is proportional to $\sigma$.Second, with the increase of saturation magnetic induction intensity $B_{m}, \frac{\Delta \mu}{\mu}$ will change in proportion.Third, With the increase of saturation magnetostrictive coefficient $\lambda_{m}, \frac{\Delta \mu}{\mu}$ will change in proportion.

\section{Simulation Analysis of Magnetic Coupling Effect of Pipeline Defects Based on ANSYS}

Finite Element Analysis Model. By using ANSYS finite element analysis software, a model of ferromagnetic defect pipeline is established. According to the force magnetic coupling model, and the simulation of the loading conditions under the action of magnetic field and external stress, the change rule of leakage magnetic field in the stress concentration area of the pipeline is discussed under different external stress conditions. An X60 military pipeline with a length of $400 \mathrm{~mm}$ is used as a model. The outside diameter of pipe is $79.5 \mathrm{~mm}$, and inside diameter is $7.2 \mathrm{~mm}$. The three different types of defects, such as cracks, pits and holes, are produced by the position of the pipeline in the middle of the pipeline.

Finite element analysis of stress field of pipeline defects. The pipeline model is established by using SOLID45 unit. The modulus of elasticity $E$ is set to $2.05 \times 10^{5} \mathrm{MPa}$, and Poisson ratio $\sigma$ is set to 0.3 . In order to save the computer hardware resource and reduce the solution time, only the $1 / 2$ model of the pipeline is established. After the careful division of the grid in the vicinity of the defect, the degree of freedom constraint is imposed at the end of the pipe, to limit its degrees of freedom along the $\mathrm{X}$ axis, the $\mathrm{Y}$ axis and the $\mathrm{Z}$ axis. At the other end of the pipe, the axial stress of different sizes is applied.

Finite element analysis of the leakage magnetic field of the pipeline's defects. A thin layer air model is established, and the model is made of OVERLAP operation between $410 \times 180 \times 100 \mathrm{~mm}$ cube and pipe model. In order to simulate the magnetic field of the magnetic field, the 0.5Gauss constant magnetic field is applied as the load of the whole model.

\section{The Relationship between Geometrical Parameters of Pipeline Defects and Leakage Magnetic Field}

The observation index of the test is the peak to peak value of the normal intensity curve of the defect leakage magnetic field. Through the experiment, the relationship between the leakage magnetic field normal strength curve peak to peak value and the parameters of crack, through hole and pit is discovered, shown in Fig. 2 - Fig. 7.

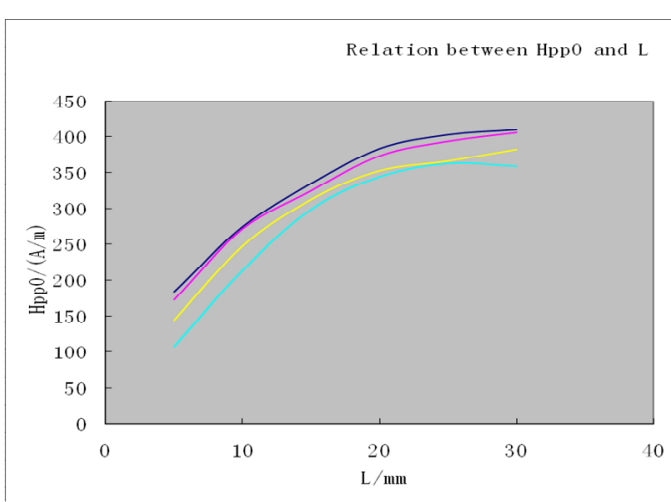

(a)

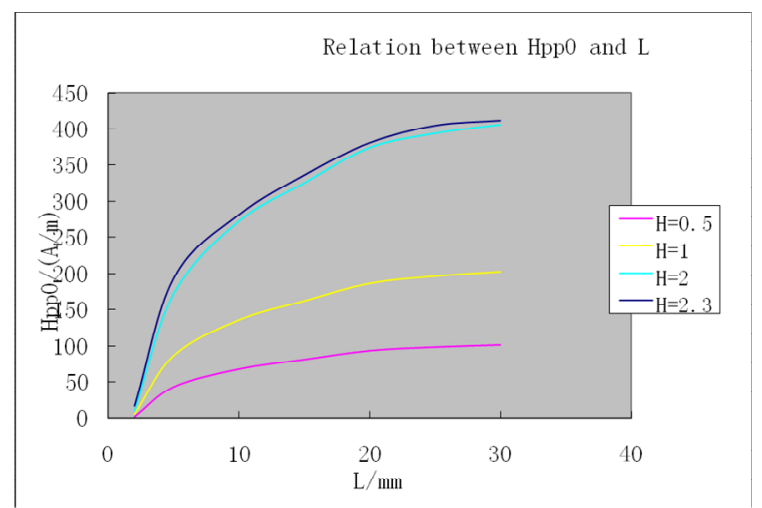

(b)

Fig. 2 Relationship between crack length $\mathrm{L}$ and $H_{p p 0}$. 
As shown in Fig. 2, the relationship between $H_{p p 0}$ and the crack length is parabolic. After the crack length is more than $25 \mathrm{~mm}$, the $H_{p p 0}$ grows slowly. When the crack width is smaller, the effect of crack width on $H_{p p 0}$ is negligible. But when the width exceeds a certain value, the $H_{p p 0}$ reduces. Within a certain range, $H_{p p 0}$ increases with the increase of the depth of the crack defects. When the depth increases to a certain value, the increase of $H_{p p 0}$ is not obvious.

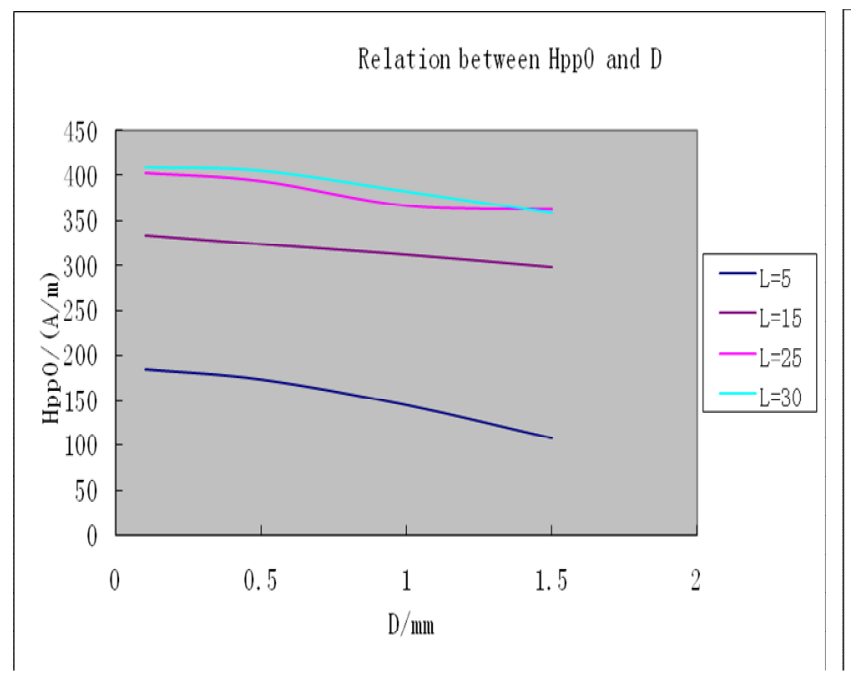

(a)

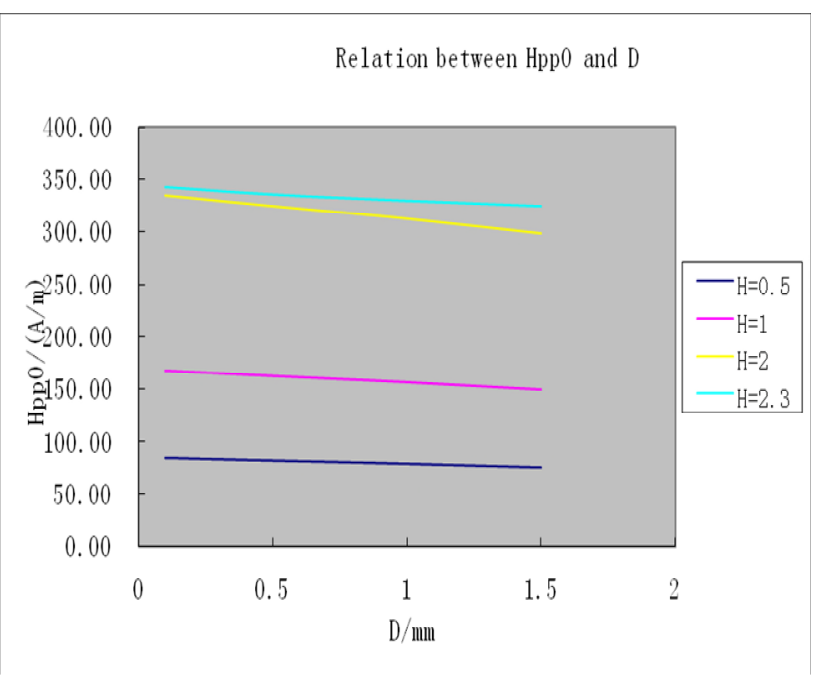

(b)

Fig. 3 Relationship between crack width D and $H_{p p 0}$.

As shown in Fig. 3, when the crack width is very small, it has less influence on the magnetic flux leakage field. But when the width of is more than $0.5 \mathrm{~mm}, H_{p p 0}$ will reduce.

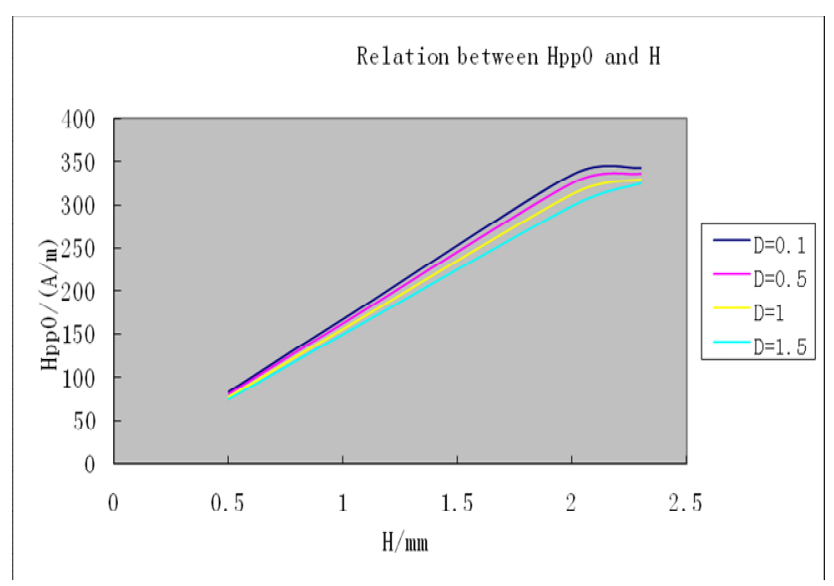

(a)

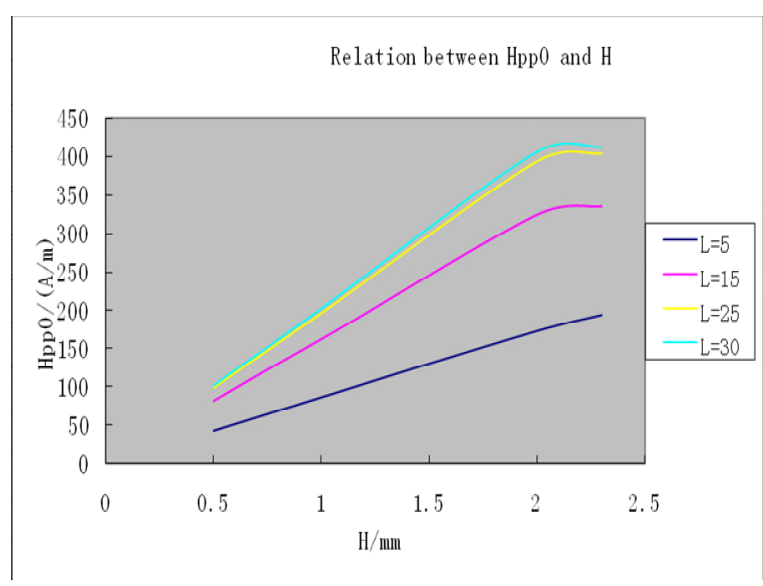

(b)

Fig. 4 Relationship between crack depth $\mathrm{H}$ and $H_{p p 0}$.

As shown in Fig. 4, when the crack depth is between 0 to $2 \mathrm{~mm}$, with the increase of the crack depth, the relationship between $H_{p p 0}$ and depth is approximately linear increase. After the crack depth is more than $2 \mathrm{~mm}$, the change of $H_{p p 0}$ is located in the nonlinear region, the growth is slow, and tends to be gentle. 


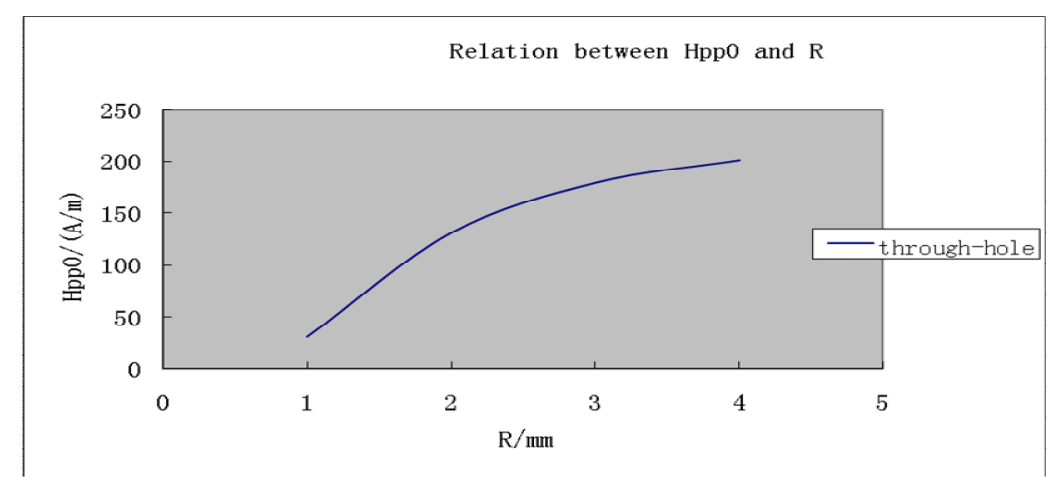

Fig. 5 Relationship between through-hole radius $\mathrm{R}$ and $H_{p p 0}$.

As shown in Fig. 5, the relationship between $H_{p p 0}$ and the radius $\mathrm{R}$ of the through hole is parabolic. When the radius is more than $3 \mathrm{~mm}$, the $H_{p p 0}$ increases slowly.
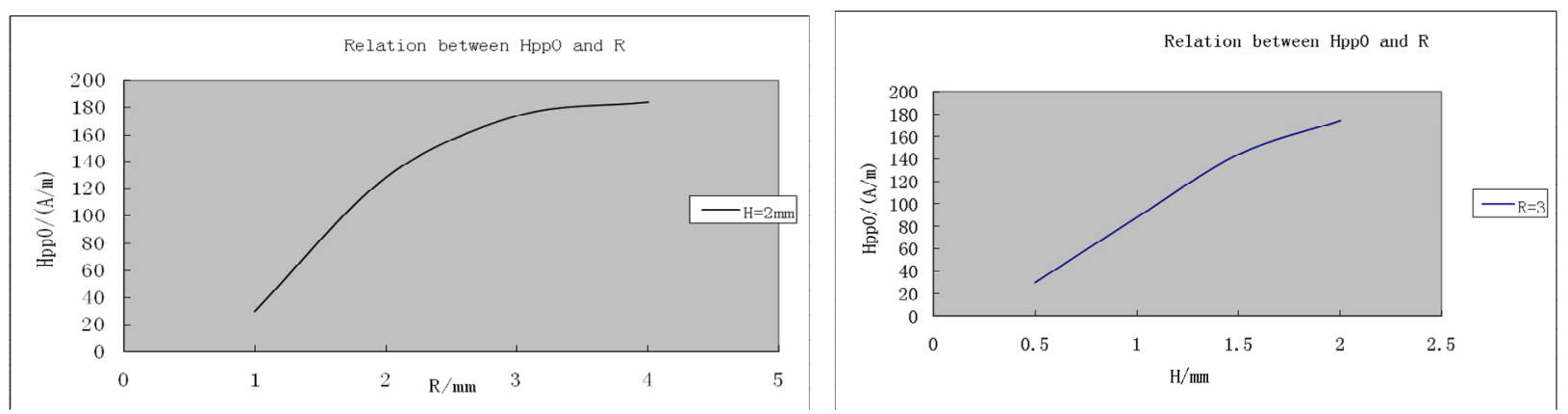

Fig. 6 Relation between dimple radius R and $H_{p p 0}$ Fig.7. Relation between dimple depth $\mathrm{H}$ and $H_{p p 0}$.

As shown in Fig. 6, the relationship between $H_{p p 0}$ and $\mathrm{R}$ is a parabola. When $\mathrm{R}$ is greater than 3 $\mathrm{mm}$, the growth of $H_{p p 0}$ becomes slow.

As shown in Fig. 7, the relationship between $H_{p p 0}$ and the pit depth $\mathrm{H}$ is approximately linear. This is consistent with the change of the leakage magnetic field in the stress concentration area of the pipeline under the condition of external stress.

\section{Conclusion}

In the paper, by establishing the mode of force magnetic coupling model and the application of ANSYS finite element analysis software, the relationship between the three kinds of defect parameters, such as pipe crack, pit and through hole, is studied systematically. The law between stress concentration and magnetic memory effect is verified.

\section{Acknowledgements}

This work was financially supported by the Postdoctoral Science Foundation.

\section{References}

[1] A. A. Doubov. A study of metal properties using the method of magnetic memory, Metal Sci. Heat Treatment, 39(9/10) (1997) 401-405.

[2] A. A. Doubov, The express technique of welded joints examination with use of metal magnetic memory, NDT \& E International, 33(6) (1997) 351-362. 
[3] A. A. Doubov. Diagnostics of metal and equipment by means of metal magnetic memory, Proceedings of 7th Conference on NDT and International Research Symposium. Shantou China, (1999), pp. 181-187.

[4] J. L. Ren, G. H. Wu, K. Song, Study on the mechanism of metal magnetic memory testing, Nonde Structive Testing, 24(1) (2002) 29-31.

[5] W. D. Zhong. Iron magnetism, Beijing: Science Press, (1998), pp. 25-38.

[6] S. L. Wang, W. Wang, S. Q. Su, S. F. Zhang, A magneto-mechanical model on differential permeability and stress of ferromagnetic material, J. Xi'an Univ. Sci. Tech. 25(3) (2005) 288-291. 